\title{
Infecciones del tracto genital y urinario como factores de riesgo para parto pretérmino en adolescentes
}

\author{
Diana Ugalde-Valencia ${ }^{1}$, María Guadalupe Hernández-Juárez ${ }^{2}$, Martha Adriana Ruiz- \\ Rodríguez ${ }^{3}$, Enrique Villarreal-Ríos. 4 \\ ${ }^{1}$ Unidad de Medicina Familiar N 10 Querétaro, ${ }^{2}$ Unidad de Medicina Familiar N5 Querétaro, ${ }^{3}$ Unidad de Medicina Fa- \\ miliar N² Querétaro, ${ }^{\circ}$ Unidad de Investigación Epidemiológica y en Servicios de Salud, Querétaro; Instituto Mexicano \\ del Seguro Social (IMSS).
}

\section{RESUMEN}

Objetivo: Determinar las infecciones genitourinarias como factores de riesgo para parto pretérmino en adolescentes. Método: Estudio de casos y controles en las Unidades de Medicina Familiar del IMSS Querétaro, periodo octubre 2010 a octubre 2011 . Se incluyeron 35 adolescentes con parto pretérmino y 148 con parto a término, mediante muestreo no aleatorio por cuota; se excluyeron aquellas con preeclampsia, eclampsia u otras enfermedades. Los datos se recolectaron del expediente clínico electrónico. Se estudiaron variables sociodemográficas; antecedentes gineco-obstétricos; tipo de parto (pretérmino y término); e infecciones urinarias y vaginales. La infección de vías urinarias fue diagnosticada mediante urocultivo y/o examen general de orina, con $\geq 10^{5}$ UFC y $>10$ leucocitos por campo, respectivamente. La infección vaginal se diagnosticó por exudado vaginal con reporte de patógenos y/o por clínica. El análisis se realizó con porcentajes, promedios, chi2, razón de momios (Odds Ratio), prueba de t e intervalo de confianza. Resultados: De las adolescentes con parto pretérmino el $54,3 \%$ presentaron infección de vías urinarias, mientras que solo $33,8 \%$ de estas presentaron parto a término $(p=0,02)$. El $57,1 \%$ de las adolescentes con parto pretérmino presentaron infección vaginal en comparación con $35,1 \%$ de las de parto a término $(p=0,01)$. Conclusión: Las infecciones vaginales y urinarias incrementan dos veces el riesgo de presentar parto pretérmino en adolescentes.

\section{PALABRAS CLAVE: Infecciones vaginales, infecciones urinarias, parto pretérmino, adolescentes}

\section{SUMMARY}

Objective: To determine genitourinary infections as risk factors for preterm delivery in teenagers. Method: Case-control study in the Family Medicine Unit IMSS Querétaro, from October 2010 to October 2011. We included 35 teenagers with preterm delivery and 148 who delivered at term, using non-random sampling, we excluded those with preeclampsia, eclampsia or other diseases. Data were collected from electronic medical record. We studied socio-demographic variables, gyneco-obstetrics antecedents, mode of delivery (preterm and term), and genitourinary infections. Urinary tract infection was diagnosed by urine culture or urinalysis, with $\geq 10^{5} \mathrm{CFU}$ and $>10$ leukocytes per field, respectively. Vaginal infection was diagnosed by vaginal culture with report of pathogens and / or by clinic. The analysis was performed using percentages, averages, chi-squared test, odds ratio, t-test and confidence intervals. Results: The $54.3 \%$ of the teenagers with preterm delivery had urinary tract infection, while only $33.8 \%$ of those with term delivery presented it $(p$ $=0.02$ ). Also $57.1 \%$ of adolescents with vaginal infection had preterm delivery compared with $35.1 \%$ of those 
giving birth at term $(p=0.01)$. Conclusion: Vaginal and urinary infections increase twice the risk of preterm delivery in teenagers.

\section{KEY WORDS: Vaginal infection, urinary tract infection, preterm delivery, teenagers}

\section{INTRODUCCIÓN}

Según la Organización Mundial de la Salud (OMS), parto pretérmino es el que ocurre antes de la semana 37 de gestación $(1,2)$. A nivel mundial la incidencia de niños prematuros varía de 5 a $10 \%$, con cifras aún mayores, en grupos como adolescentes con $21,3 \%(3,4)$. La OMS define la adolescencia como el período comprendido entre los 10-19 años (5). El riesgo relativo de prematuridad entre adolescentes, comparadas con mujeres adultas es de 1,4 a 2,0 veces mayor (4).

Las infecciones vaginales se caracterizan por síntomas como flujo, prurito vulvar, ardor, irritación, dispareunia y mal olor vaginal, por invasión de patógenos como resultado de un desbalance en el ecosistema vaginal. Son responsables del $31 \%$ al $47,9 \%$ de los partos pretérmino (6-8).

La infección urinaria es la presencia de microorganismos patogénicos en el tracto urinario. Se diagnostica mediante urocultivo con una cuenta $\geq 10^{5}$ unidades formadoras de colonias (UFC) o por examen general de orina con más de 10 leucocitos por campo (9). El $30,2 \%$ a $52,8 \%$ de los partos pretérmino son debido a infecciones urinarias (10-12).

El objetivo de este estudio es determinar si las infecciones genitourinarias son factores de riesgo para parto pretérmino en adolescentes.

\section{PACIENTES Y MÉTODO}

Se realizó un estudio de casos y controles, en adolescentes derechohabientes del IMSS que fueron atendidas por parto, de las Unidades de Medicina Familiar 2, 5 y 10 de Querétaro, en el periodo de octubre del 2010 a octubre del 2011. Se formaron dos grupos de estudio, el primero comprendido por pacientes adolescentes que presentaron parto pretérmino y el segundo por pacientes adolescentes que presentaron parto a término. Se incluyeron pacientes con hojas de control prenatal con la información necesaria para la realización de la investigación y aquellas que contaron con consulta posterior a la atención del parto. Se excluyeron aquellas que cursaron con preeclampsia, eclampsia, diabetes gestacional, entre otras enfermedades; también fueron eliminadas las pacientes cuyas hojas de control prenatal no contaban con los datos suficientes para la realización de la investigación.

El tamaño de la muestra se calculó con la fórmula de porcentajes para dos poblaciones con nivel de confianza del $95 \%$, poder de la prueba del $80 \%$ y la hipótesis de que la infección de vías urinarias estaba presente en más del $30 \%$ de las adolescentes con parto pretérmino y en más del $15 \%$ de las adolescentes con parto a término. Obteniendo un número de 93 pacientes en cada grupo. Sin embargo, el total de la población estudiada fue de 35 pacientes con parto pretérmino y 148 con parto a término, esto permite trabajar con un nivel de confianza del $86 \%$ con una relación de 1 a 4 para el tamaño de la muestra.

La técnica muestral fue no aleatoria por cuota, utilizando como marco muestral el listado de adolescentes embarazadas proporcionado por el departamento de archivo de cada una de las unidades de medicina familiar. Para la recolección de datos se revisó el expediente clínico electrónico de cada una de las pacientes seleccionadas para el estudio.

Las variables estudiadas fueron sociodemográficas (edad y escolaridad); antecedentes ginecoobstétricos (menarca, edad de inicio de vida sexual activa, gestas, partos, cesáreas y abortos); tipo de parto (pretérmino y a término); y la infección de vías urinarias o vaginales.

El parto pretérmino fue definido como aquel que ocurre entre las 20 y 37 semanas de gestación. El parto a término es el que ocurre entre las 38 y 42 semanas de gestación. Esto verificando la fecha de última menstruación (FUM) y las semanas de gestación al momento del parto en el expediente.

La infección de vías urinarias se diagnosticó por urocultivo o examen general de orina, al encontrarse una cuenta $\geq 10^{5}$ unidades formadoras de colonias (UFC) y más de 10 leucocitos por campo, respectivamente. La infección vaginal es definida como la invasión y multiplicación de agentes microbianos en la vagina. El diagnóstico se realizó por exudado vaginal con reporte de aislamiento de microorganismos patógenos y/o por clínica.

El análisis estadístico se llevó a cabo con porcentajes, promedios, prueba de chi2, razón de momios (Odds Ratio), prueba de t e intervalo de confianza, empleando el programa estadístico SPSS. 


\section{RESULTADOS}

La edad promedio de adolescentes con parto pretérmino y con parto a término fue de 18,31 años y 18,23 años, respectivamente. Los antecedentes gineco-obstétricos no mostraron diferencias estadísticamente significativas en ambos grupos (Tabla I).

La escolaridad que predominó en las adolescentes fue secundaria, obteniéndose en un $65,7 \%$ de aquellas con parto pretérmino y un $64,2 \%$ de las de parto a término (Tabla II).

El $54,3 \%$ de las adolescentes con parto pretérmino presentaron infección de vías urinarias, mientras que el $33,8 \%$ de estas presentaron parto a término $(p=0,02)$. La infección vaginal estuvo presente en el $57,1 \%$ de las adolescentes con parto pretérmino en comparación con el $35,1 \%$ presente en aquellas con parto a término $(p=0,01)$ (Tabla III).

Tabla I

EDAD Y ANTECEDENTES GINECO-OBSTÉTRICOS DE PACIENTES CON PARTO PRETÉRMINO Y DE TÉRMINO

\begin{tabular}{lcccccc}
\hline & \multicolumn{2}{c}{ Pretérmino } & \multicolumn{2}{c}{ Término } & & \\
Variable & Promedio & $\begin{array}{c}\text { Desviación } \\
\text { estándar }\end{array}$ & Promedio & $\begin{array}{c}\text { Desviación } \\
\text { estándar }\end{array}$ & $\mathrm{t}$ & Valor $\mathrm{p}$ \\
\hline Edad & 18,31 & 0,91 & 18,23 & 1,23 & 0,61 & 0,54 \\
Menarca & 12,62 & 1,23 & 12,31 & 1,24 & 1,36 & 0,17 \\
Inicio de vida sexual activa & 16,91 & 1,29 & 16,47 & 1,54 & 1,54 & 0,12 \\
Gestas & 1,28 & 0,51 & 1,20 & 0,45 & 0,86 & 0,38 \\
Partos & 0,08 & 0,28 & 0,08 & 0,27 & 0,08 & 0,92 \\
Cesáreas & 0,08 & 0,28 & 0,06 & 0,23 & 0,53 & 0,59 \\
Abortos & 0,11 & 0,32 & 0,06 & 0,27 & 0,58 & 0,38 \\
\hline
\end{tabular}

Tabla II

ASOCIACIÓN DE ESCOLARIDAD CON PARTO PRETÉRMINO Y DE TÉRMINO

\begin{tabular}{lcccc}
\hline & \multicolumn{2}{c}{ Porcentaje } & Chi cuadrada & Valor $p$ \\
Variable & Pretérmino & Término & & \\
\hline Sabe leer y escribir & 0,0 & 1,4 & & \\
Primaria & 14,3 & 13,5 & 0,63 & 0,95 \\
Secundaria & 65,7 & 64,2 & & \\
Preparatoria & 17,1 & 18,9 & & \\
Profesional & 2,9 & 2,0 & & \\
\hline
\end{tabular}

Tabla III

ASOCIACIÓN DE LA INFECCIÓN DE VÍAS URINARIAS Y LA INFECCIÓN VAGINAL CON PARTO PRETÉRMINO Y DE TÉRMINO

\begin{tabular}{lccccccc}
\hline & \multicolumn{2}{c}{ Porcentaje } & Chi & Valor & Razón & \multicolumn{2}{c}{ Intervalo de confianza } \\
Variable & Pretérmino & Término & cuadrada & $\mathrm{p}$ & momios & Inferior & Superior \\
\hline $\begin{array}{l}\text { Infección de vías } \\
\text { urinarias }\end{array}$ & 54,3 & 33,8 & 5,06 & 0,02 & 2,32 & 1,10 & 4,91 \\
\begin{tabular}{l} 
Infección vaginal \\
\hline
\end{tabular} & 57,1 & 35,1 & 5,74 & 0,01 & 2,46 & 1,16 & 5,21 \\
\hline
\end{tabular}




\section{DISCUSIÓN}

Nuestro estudio confirma que la infección de vías urinarias y la infección vaginal se asocian significativamente con un mayor riesgo de parto prematuro en una población de embarazadas adolescentes.

Respecto a las variables sociodemográficas (edad y escolaridad) no encontramos diferencia significativa entre los grupos, puesto que la edad promedio de las adolescentes con parto pretérmino fue de 18,31 años, mientras que para aquellas con parto a término fue de $18,23(p=0,54)$. Además en ambos grupos predominó la escolaridad secundaria; contrario a lo encontrado en la literatura, que reporta que el nivel cultural, valorado como grado de escolaridad, ha sido relacionado como factor de riesgo, pues está estrechamente ligado a la ocupación (que a su vez se asocia con el grado de actividad física durante el embarazo) así como al nivel socioeconómico y de acceso a los servicios médicos.

En relación a los antecedentes gineco-obstétricos (menarca, inicio de vida sexual activa, gestas, partos, cesáreas y abortos), los resultados obtenidos muestran que ninguno de éstos son representativos para el tipo de parto en las adolescentes, acorde a lo reportado en la literatura, puesto que el simple hecho de encontrarse dentro de este rango de edad representa la inclusión al más importante riesgo para el parto pretérmino, dato que no es posible encontrar en este estudio, dado que todas las pacientes que formaron parte de la muestra se encuentran dentro del mismo grupo de edad.

Las infecciones vaginales se encuentran presentes en el $57,1 \%$ de las adolescentes con parto pretérmino, similar a la literatura, que reporta que son responsables del $31 \%$ al $47,9 \%$ de los partos pretérmino. El antecedente de infección vaginal incrementa 2,46 veces el riesgo de presentar parto pretérmino $(p=0,01)$, puesto que la exposición a patógenos vaginales durante el embarazo desencadena un proceso inflamatorio que a su vez provoca el trabajo de parto o la rotura prematura de las membranas.

Las infecciones en vías urinarias, presentan un comportamiento similar a las infecciones vaginales, con una incidencia de $54,3 \%$ en las adolescentes con parto pretérmino, contra un $38,8 \%$ en aquellas con parto a término. Lo cual también se relaciona con lo reportado en la literatura, que informa que hasta el $52,8 \%$ de los partos pretérmino son debido a infecciones de vías urinarias. Así mismo, la infección urinaria incrementa el riesgo de parto pretérmino 2,32 veces $(p=0,02)$.

\section{CONCLUSIÓN}

Las infecciones de vías urinarias así como las infecciones vaginales constituyen un factor de riesgo para el parto pretérmino en la embarazada adolescente, por ello se propone la instrucción adecuada del personal de salud para la detección oportuna y el tratamiento adecuado de las infecciones genitourinarias para disminuir la tasa de morbilidad y mortalidad neonatal secundario a prematurez.

\section{REFERENCIAS}

1. Calderón J, Vega G, Velázquez J, Morales R, Vega AJ. Factores de riesgo materno asociados al parto pretérmino. Rev Med IMSS 2005;43:339-42.

2. Debiec KE, Paul KJ, Mitchell CM, Hitti JE. Inadequate prenatal care and risk of preterm delivery among adolescents: a retrospective study over 10 years. Am J Obstet Gynecol 2010;203:1-6.

3. Althabe F, Carroli G, Lede R, Belizán JM, Althabe $\mathrm{OH}$. El parto pretérmino: detección de riesgos y tratamientos preventivos. Rev Panam Salud Publica /Pan Am J Public Health 1999;5:373-85.

4. Villanueva LA, Contreras AK, Pichardo M, Rosales J. Perfil epidemiológico del parto prematuro. Ginecol Obstet Mex 2008;76:542-8.

5. Manrique R, Rivero A, Ortunio M, Rivas M, Cardozo $\mathrm{R}$, Guevara H. Parto pretérmino en adolescentes. Rev Obstet Ginecol Venez 2008;68:141-9.

6. Oviedo H, Lira J, Ito A, Grosso JM. Causas de nacimiento pretérmino entre madres adolescentes. Ginecol Obstet Mex 2007;75:17-23.

7. Cires M, Freijoso E, Silva L, Vergara E, Cutié E, Ortega $\mathrm{M}$, et al. Guía para la práctica clínica de las infecciones vaginales. Rev Cubana Farm 2003;37:38-52.

8. Lamont RF. Antibiotics used in women at risk of preterm birth. Am J Obstet Gynecol 2008;7:583-4.

9. Ruiz G. Padecimientos infecciosos: Infecciones del tracto genito-urinario. En: Fundamentos de interpretación clínica de los exámenes de laboratorio. $1^{\text {a }}$ edición. México: Editorial Panamericana, 2005;161-3.

10. Kurnit K, Cahill A, Jeffcoat M, Parry S, Sammel M, Nelson $\mathrm{D}$, et al. Infections in pregnancy: synergistic effect on spontaneous preterm delivery (SPTD) risk? Am J Obstet Gynecol 2008;199:57-8.

11. Bojanini JF, Gómez JG. Resultados obstétricos y perinatales en adolescentes. Rev Col Obstet Ginecol 2004;55:114-21.

12. Vázquez A, Guerra C, Herrera V, De la Cruz F, Almirall AM. Embarazo y adolescencia: factores biológicos materno y perinatal más frecuentes. Rev Cubana Obstet Ginecol 2001;27:158-64. 